\title{
Temporal scaling of groundwater discharge in dual and multicontinuum catchment models
}

\author{
Anna Russian, ${ }^{1}$ Marco Dentz, ${ }^{2,3}$ Tanguy Le Borgne, ${ }^{4}$ Jesus Carrera, ${ }^{2,3}$ and Joaquin Jimenez-Martinez ${ }^{4}$ \\ Received 12 June 2013; revised 11 November 2013; accepted 24 November 2013; published 19 December 2013.
}

[1] This paper presents a multicontinuum approach to model fractal temporal scaling of catchment response in hydrological systems. The temporal scaling of discharge is quantified in frequency domain by the transfer function $\Theta(\omega)$, which is defined as the ratio between the spectra of catchment response and recharge time series. The transfer function may scale with frequency $\omega$ as $\Theta(\omega) \sim \omega^{-\beta}$. While the classical linear and Dupuit models predict exponents of $\beta=2$ and $\beta=1$, observations indicate scalings with noninteger exponents $\beta$. Such behaviors have been described by multifractal models, which, however, often lack a relation to the medium characteristics. We revisit and extend the classical linear Dupuit aquifer models and discuss their physical meanings in the light of the resulting aquifer dynamics. On the basis of these classical models, we derive a multicontinuum approach that provides physical recharge models which are able to explain fractal behaviors with exponents $1 / 2<\beta<2$. Furthermore, this approach allows to link the fractal dynamics of the discharge process to the physical aquifer characteristics as reflected in the distribution of storage time scales. We systematically analyze the catchment responses in the proposed multicontinuum models, and identify and quantify the time scales which characterize the dynamics of the catchment response to recharge.

Citation: Russian, A., M. Dentz, T. Le Borgne, J. Carrera, and J. Jimenez-Martinez (2013), Temporal scaling of groundwater discharge in dual and multicontinuum catchment models, Water Resour. Res., 49, 8552-8564, doi:10.1002/2013WR014255.

\section{Introduction}

[2] The understanding of the relation between aquifer discharge and recharge is a fundamental problem in hydrology. Often, aquifer discharge into a river or any outfall is the only information available when studying the groundwater system of a basin. This information may be enough to model regional flow, disregarding the details at local scales [Duffy and Lee, 1992]. The discharge of a catchment is a measure for the recharge at basin-scale, and variations in the discharge represent the dynamic response of an aquifer to changes in recharge. A clear understanding of the groundwater system dynamics and the recharge processes is fundamental to improve our ability to manage groundwater resources. The ability to understand and model hydrological processes at basin scale is fundamental to improve our ability to manage groundwater resources and predict the effect of large-scale changes in land use and climate.

[3] Modeling aquifer discharge is a challenging problem because the discharge of a catchment after a rainfall event

\footnotetext{
${ }^{1}$ Géosciences, Université de Montpellier 2, CNRS, Montpellier, France. ${ }^{2} \mathrm{GHS}$, Institute of Environmental Assessment and Water Research (IDAEA), Spanish National Research Council (CSIC), Barcelona, Spain.

${ }^{3}$ GHS, Technical University of Catalonia (UPC), Barcelona, Spain.

${ }^{4}$ Géosciences Rennes, CNRS, Université de Rennes 1, Rennes, France.

Corresponding author: A. Russian, Géosciences, Université de Montpellier 2, CNRS, Place E. Bataillon, Montpellier FR-34095, France. (anna.russian@yahoo.it)
}

(C)2013. American Geophysical Union. All Rights Reserved. 0043-1397/13/10.1002/2013WR014255 is the result of several processes, such as rainfall infiltration, surface detention, overland flow, surface runoff, lateral and preferential flow, transient saturated and unsaturated flow [Scanlon et al., 2002; Fiori and Russo, 2007]. Information on these processes is typically limited. The main sources of complexity are: (i) the nonlinearity of many hydrological process and (ii) the high degree of variability of conditions in space and time [Dingman, 1994]. Fiori and Russo [2007] show using numerical simulations that the spatial variability of hydraulic parameters has a noteworthy impact on water movement and catchment response.

[4] Spatial heterogeneity brings complexity in the flow dynamics and induces uncertainty. Even if it was possible to have a detailed description of heterogeneity, the amount of information would be difficult to handle and the resulting basin-scale dynamics difficult to interpret. Because of the scarcity of information and the intrinsic complexity in the recharge process, it is useful to keep basin-scale models simple, and search for a description of the basin-scale aquifer dynamics that integrates the collective action of heterogeneity and small-scale flow processes. For these reasons, we focus on the impact of spatial heterogeneity on the catchment response at hillslope scale starting from a linearized classical recharge model, the linear Dupuit model [Gelhar and Wilson, 1974]. Spatial heterogeneity of hydraulic parameters is handled stochastically. Temporal variability in the recharge process is conveniently handled in spectral domain [e.g., Gelhar, 1974; Besbes and De Marsily, 1984; Duffy and Cusumano, 1998; Manga, 1999]. We consider a spatially uniform recharge, absence of return 
$r(\mathrm{t})$

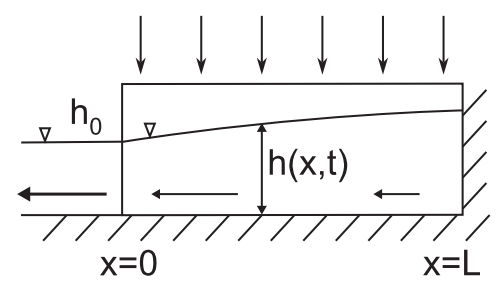

$r(t)$

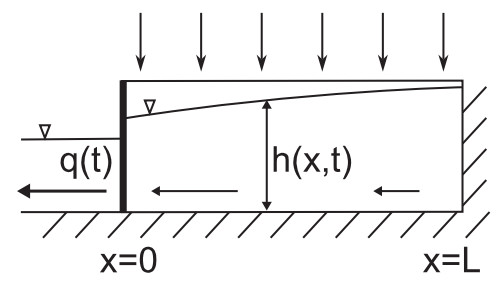

$r(t)$

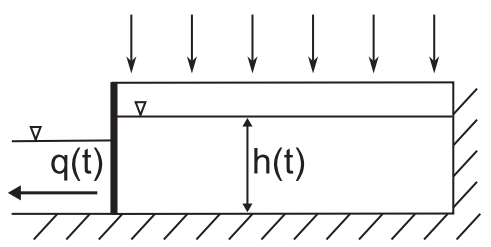

Figure 1. Sketch of the classical recharge models. From left to right, Linear Dupuit (LD) model with Dirichlet BC, Dupuit model with Cauchy BC, and the Linear Reservoir (LR) model. $q(t)$ denotes stream discharge, $r(t)$ aquifer recharge (assumed spatially uniform), $h(t)$ and $h(x, t)$ hydraulic head, $L$ width of the catchment. $h_{0}$ indicates the boundary head at the outfall for the LD model with Dirichlet BC. In the LR model and in the LD model with Cauchy BC, $q(t)$ indicates the flux at the outfall. In the Dupuit models at $x=L$ no-flux BC at the watershed is imposed.

flow and overland flow, which are reasonable assumptions especially for a small catchment [Dingman, 1994].

[5] The simplest way to model the catchment response is to consider the catchment as a linear input-output system. Input (recharge) and the output (groundwater head variation or the consequent discharge) are related linearly through a transfer function, which quantifies the action of the aquifer on the discharge dynamics. In this context, the aquifer is considered a linear filter whose properties depend on the medium characteristics and flow processes in the medium [e.g., Gelhar, 1974; Freeze, 1975; Duffy and Gelhar, 1985; Juki and Denijuki, 2004; Zhang, 2004]. Spectral analysis provides a useful tool to characterize the input and output signals and analyze the filter properties of the aquifer [e.g., Gelhar, 1974]. In this framework, the aquifer dynamics are quantified by the power spectrum of the transfer function, which in the following is termed the frequency transfer function $\Theta(\omega)$. The frequency transfer function (FTF) reflects the action of the aquifer on the head and discharge dynamics.

[6] Classical physical aquifer models to determine the transfer function are the linear reservoir (LR) model and the linear Dupuit (LD) aquifer model [e.g., Gelhar, 1974]. The LR model is zero-dimensional and thus disregards spatial variations of hydraulic head. The LD model is onedimensional and describes flow in the aquifer based on the linearized Dupuit-Forchheimer hypothesis [e.g., Bear, 1972]. These models are illustrated schematically in Figure 1. They predict that for high frequencies $\omega$, the transfer function related to the discharge flux scales as $\Theta_{q}(\omega) \sim \omega^{-\beta}$. The LR model predicts a scaling exponent of $\beta=2$ [Gelhar, 1974], the LD model a scaling exponent of $\beta$ $=1$ [Gelhar, 1974; Molenat et al., 1999]. The difference in the scaling exponents implies a difference in the aquifer response dynamics.

[7] A series of studies have shown that groundwater levels and discharge signals [e.g., Zhang and Yang, 2010; Labat et al., 2002; Zhang, 2004; Molenat et al., 1999, 2000; Jiménez-Martínez et al., 2013] as well as river runoff [e.g., Tessier et al., 1996; Kantelhardt et al., 2003; Zhang, 2005; Kantelhardt et al., 2006; Koscielny-Bunde et al., 2006; Livina et al., 2007; Little and Bloomfield, 2010] may scale with noninteger exponents $\beta$. Such behavior cannot be explained by classical discharge models, which are based on the representation of the aquifer as a homogeneous porous medium. In fact, such behavior is commonly attributed to the heterogeneity of the catchment. Spatial heterogeneity induces a spectrum of transfer time scales, which the classical models are not able to describe. A detailed review of catchment transit time modeling is given in McGuire [2006]. Conceptually, we can imagine that after a recharge event, a portion of water may flow quickly to the discharge point according to the piezometric head gradient, whereas another portion may infiltrate in low permeability regions and get stored there to be released slowly at a later time. As indicated by Hurst [1951], long-range correlations in river responses indicate that water storage and discharge processes occur over a wide range of temporal scales [Tessier et al., 1996; Fiori et al., 2009; Duffy, 2010].

[8] Noninteger exponents of the frequency transfer function can be explained by interflow through the vadose zone [Fiori et al., 2009] or modeled on the basis of Nash transfer functions, which consider a cascade of a number of linear reservoir with identical storage coefficients [Nash, 1957; Nasri et al., 2004] or using multifractal approaches [e.g., Turcotte and Greene, 1993; Tessier et al., 1996; Kantelhardt et al., 2003; Labat et al., 2011]. A limitation of these models is that the link with a measurable property of the medium is missing. For example, the fractal dimension is not directly linked to the spatial organization of the aquifer, and it can vary depending on the experimental conditions used to determine it [e.g., Little and Bloomfield, 2010]. For these reasons, the interpretation of these models is rather difficult [Tessier et al., 1996], which limits their usefulness for prediction [Labat et al., 2002]. Zhang [2004] pointed out that scaling characterized by noninteger exponents in the aquifer response may be due to medium heterogeneity, or fractality of the recharge process, or a combination of both.

[9] In this paper, we focus on the role of medium heterogeneity for the scaling of the aquifer response to recharge. The objective is to establish a model that renders a wide range of water storage scales, that is able to explain fractal behavior and that allows to link the fractal dynamics of the discharge process to a stochastic description of the medium heterogeneity as reflected in the distribution of storage scales. To this end, we consider dual and multicontinuum aquifer models that can explain preasymptotic fractal scaling of the transfer function due to the medium heterogeneity. Early double porosity and double permeability models assume that the mobile and immobile zones are each in 
quasi-equilibrium and therefore mass transfer is approximated as a first-order process [e.g., Barenblatt et al., 1960; Warren and Root, 1963; Dykhuizen, 1987, 1990]. Our approach takes into account nonequilibrium effects in the immobile zone, which together with heterogeneity gives rise to noninteger exponents in frequency transfer functions.

[10] In the following, we revisit and extend the classical recharge models and discuss their physical meanings in the light of the resulting aquifer dynamics. On the basis of these classical models, we derive dual and multicontinuum recharge models which are able to explain noninteger exponent of the frequency transfer function spectrum and link this behavior to a statistical description of the medium heterogeneity.

\section{Classical Dupuit Model}

[11] The classical linear Dupuit (LD) model is based on the linearized form of the Dupuit approximation of the flow equation for homogeneous porous media [Bear, 1972]. It is given by [Gelhar, 1974]

$$
S \frac{\partial h(x, t)}{\partial t}-\mathrm{T} \frac{\partial^{2} h(x, t)}{\partial x^{2}}=r(t)
$$

where $S$ is storativity [-], $h(x, t)$ is hydraulic head [L], T is transmissivity $\left[\mathrm{L}^{2} \mathrm{~T}^{-1}\right], r(t)$ is recharge per unit aquifer surface area $\left[\mathrm{LT}^{-1}\right], x$ is distance from the discharge point, and $t$ is time. Per Darcy's law, the discharge per unit aquifer surface area $q(t)$, is given by

$$
q(t)=-\left.\frac{\mathrm{T}}{\mathrm{L}} \frac{\partial h(x, t)}{\partial x}\right|_{x=0}
$$

where $\mathrm{L}$ is the width of the aquifer. A schematic illustration of the model is given in Figure 1.

\subsection{Spectral Analysis}

[12] In modeling recharge dynamics as an input-output problem, linearity implies that the output signal (the groundwater level or the consequent discharge) represents a unique response to a given input (recharge). This approach assumes that the recharge $r(t)$ is spatially uniform, which is a reasonable assumption for small basins. Thus, groundwater discharge $q(t)$ can be written as a linear functional of the input signal $r(t)$,

$$
q(t)=\int_{-\infty}^{\infty} f_{q}\left(t^{\prime}\right) r\left(t-t^{\prime}\right) d t^{\prime}
$$

where $f_{q}(t)$ is the discharge impulse response function, or filter, of the system [Wiener, 1949]. Both the impulse response function $f_{q}(t)$ and recharge $r(t)$ are zero for $t<0$. This convolution transforms to a product in frequency domain. The Fourier transform here is defined by

$$
\tilde{f}(\omega)=\int_{-\infty}^{\infty} f(t) \exp (-i \omega t) d t, \quad f(t)=\int_{-\infty}^{\infty} \tilde{f}(\omega) \exp (i \omega t) \frac{d \omega}{2 \pi}
$$

with $i=\sqrt{-1}$ the imaginary unit. Here and in the following, Fourier-transformed quantities are marked by a tilde.
[13] Taking the Fourier transform of equation (3) and solving for $\tilde{f}_{q}(\omega)$, we obtain the complex frequency discharge response function $\tilde{f}_{q}(\omega)$ as the ratio of the Fourier transforms of the discharge $\tilde{q}(\omega)$ and the recharge $\tilde{r}(\omega)$. Its square defines the frequency transfer function (FTF) [Box and Jenkins, 1970]:

$$
\tilde{f}_{q}(\omega)=\frac{\tilde{q}(\omega)}{\tilde{r}(\omega)}, \quad \Theta_{q}(\omega)=\left|\tilde{f}_{q}(\omega)\right|^{2} .
$$

[14] The response of the hydraulic head $h(x, t)$ is characterized by the head FTF, $\Theta_{h}(x, \omega)$, which is defined by the square of the ratio of $\tilde{h}(x, \omega)$ and of the recharge $\tilde{r}(\omega)$,

$$
\Theta_{h}(x, \omega)=\left|\frac{\tilde{h}(x, \omega)}{\tilde{r}(\omega)}\right|^{2} .
$$

[15] The head FTF depends on the location of the observation point $x$ in the basin, while the discharge FTF $\Theta_{q}(\omega)$ characterizes the dynamics of the catchment at basin scale.

\subsection{Linear Dupuit Model With Dirichlet BC}

[16] As illustrated in Figure 1, the Dupuit model represents a homogeneous catchment with a no-flow boundary condition (BC) at the basin end (Neumann BC), $\partial h(x=L, t) / \partial x=0$. The Dirichlet BC prescribes the head at the discharge boundary at $x=0, h(x=0, t)=t)=h_{0}$ [e.g., Gelhar, 1974; Molenat et al., 1999]. Without loss of generality, we set $h_{0}=0$ in the following.

[17] With this BC, the solution of the Fourier transform of equation (1) is given by

$$
\tilde{h}(x, \omega)=\frac{\tilde{r}(\omega)}{i \omega S}\left\{1-\frac{\cosh \left[p(\omega)\left(\frac{x}{L}-1\right)\right]}{\cosh [p(\omega)]}\right\}
$$

see also Gelhar [1974]. We defined here

$$
p(\omega)=\sqrt{i \omega \tau_{L}}, \quad \tau_{L}=L^{2} S / T=\omega_{L}^{-1} .
$$

[18] The inverse of the characteristic aquifer response time $\omega_{L}=\tau_{L}^{-1}$ is referred to as aquifer response rate [Erskine and Papaioannou, 1997].

[19] The discharge FTF defined in equation (5) is obtained from equation (7) and the Fourier transform of equation (2). This yields [Molenat et al., 1999; Gelhar, 1974]

$$
\Theta_{q}(\omega)=\frac{1}{\omega \tau_{L}}|\tanh [p(\omega)]|^{2},
$$

[20] The head FTF defined by equation (6) can be directly read off expression (7) and is given by

$$
\Theta_{h}(x, \omega)=\frac{1}{\omega^{2} S^{2}}\left|1-\frac{\cosh \left[p(\omega)\left(\frac{x}{L}-1\right)\right]}{\cosh [p(\omega)]}\right|^{2} .
$$

[21] The behavior of $\Theta_{q}(\omega)$ is illustrated in Figure 2. For high frequencies, $\omega \tau_{L} \gg 1$ it scales as $\omega^{-1}$. For low frequencies, $\omega \tau_{L} \ll 1, \Theta_{q}(\omega)$ approaches one. This means that long-time components in the recharge spectrum, with frequencies lower than the aquifer response rate are not smoothed by the system and their variations reflect unaltered on the output. 


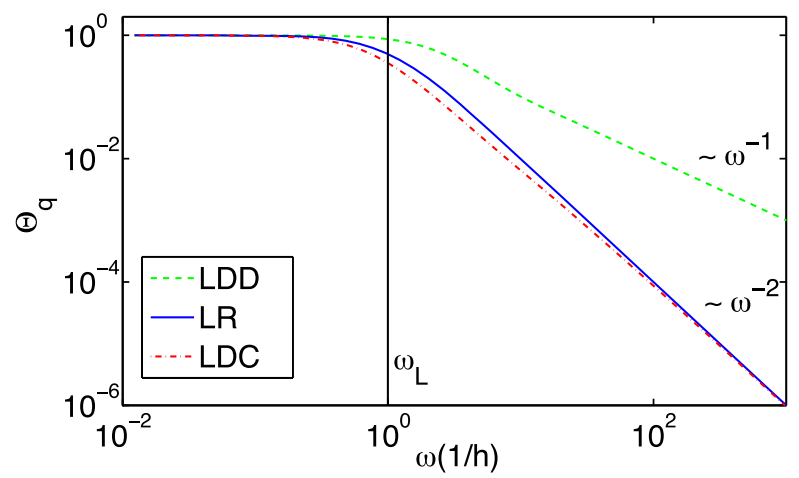

Figure 2. Discharge FTF $\Theta_{q}(\omega)$ for the (dashed) LDD, (dash-dotted) LDC, and (solid) LR models. The vertical line indicates the aquifer response rate $\omega_{L}=1 \mathrm{~h}$ which is given by $\omega_{L}=T /\left(L^{2} S\right)$ for the LDD and LDC models, and by $\omega_{L}=\alpha / S$ for the LR model. We used $S=10^{-4}, T=10^{2} \mathrm{~m}^{2} \mathrm{~h}^{-1}, L=10^{3} \mathrm{~m}$, and $\alpha=\alpha_{c}=10^{-4} \mathrm{~h}^{-1}$.

[22] The evolution of $\Theta_{h}(x, \omega)$ at $x=L, x=L / 20$, and $x=$ $L / 100$ is shown in Figure 3. Unlike the discharge FTF, the behavior of $\Theta_{h}(x, \omega)$ depends on the observation point $x$. The spatial dependence of $\Theta_{h}(x, \omega)$ introduces a second characteristic time $\tau_{x}=x^{2} S / T$, which is given by the mean diffusion time from the observation point to the discharge point. For $x / L \ll 1$, an intermediate regime develops, which is given by $\tau_{L}^{-1} \ll \omega \ll \tau_{x}^{-1}$. In this regime, the head FTF scales as the discharge FTF according to $\omega^{-1}$, which can be derived by expanding the hyperbolic cosine in equation (10) for $x / L \ll 1$. In fact, near the discharge, for $x / L \ll 1$, the discharge flux can be approximated by

$$
q(0, t) \approx-T h(x, t) /(x L)
$$

[23] For frequencies $\omega \gg \tau_{x}^{-1}$ the head FTF scales as $\omega^{-2}$. At the watershed boundary for $x=L$, it behaves exactly as $\Theta_{h}(L, \omega)=1 /\left(S^{2} \omega\right)$, see equation (10). This scaling corresponds to the behavior of the classical linear reservoir (LR) model, which is a zero-dimensional model that represents the catchment as a lumped reservoir [Gelhar, 1974], see also Appendix A.

[24] The values of $\Theta_{h}(x, \omega)$ at $\omega=0$ depend on the observation point. We obtain by taking the limit $\omega \rightarrow 0$ in equation (10)

$$
\lim _{\omega \rightarrow 0} \Theta_{h}(x, \omega)=\left|-\frac{x^{2}}{2 T}+\frac{L x}{T}\right|^{2}
$$

\subsection{Linear Dupuit Model With Cauchy BC}

[25] Dirichlet discharge boundary conditions may not always be realistic and in many situation Cauchy BCs may be more appropriate [e.g., Bear, 1972]. The Cauchy boundary condition relates the discharge flux with the difference of the boundary head and a reference head $h_{0}$ at the outlet as

$$
\left.\frac{T}{L} \frac{\partial h(x, t)}{\partial x}\right|_{x=0}=\alpha_{c}\left[h(0, t)-h_{0}\right]
$$

where $\alpha_{c}$ is an outflow constant (leakage coefficient). Without loss of generality, the reference level is set to zero. The
LD model with Cauchy boundary conditions in the following is referred to as LDC model. Note that the Cauchy boundary condition coincides with the Dirichlet condition in the limit of $\alpha_{c} \rightarrow \infty$. With this BC, the solution of the Fourier transform of equation (1) is given by

$$
\tilde{h}(x, \omega)=\frac{R(\omega)}{i \omega S}\left\{1-\frac{\cosh \left[p(\omega)\left(\frac{x}{L}-1\right)\right]}{\frac{p(\omega) T}{\alpha_{c} L^{2}} \sinh [p(\omega)]+\cosh [p(\omega)]}\right\},
$$

with $p(\omega)$ given by equation (8). Note that for $p(\omega) T / L^{2} \ll \alpha_{c}$, equation (14) reduces to equation (7). This implies that in the time regime $t \gg(S T) /\left(\alpha_{c} L\right)^{2}$ the dynamics of the LDD and LDC models are the same.

[26] Using equation (14) in the Fourier transform of equation (2) gives $\tilde{q}(\omega)$, and from equation (5) we then obtain for the discharge frequency transfer function

$$
\Theta_{q}(\omega)=\frac{\alpha_{c}^{2}}{\omega^{2} S^{2}}\left|\frac{\tanh [p(\omega)]}{\tanh [p(\omega)]+\frac{\alpha_{c} L^{2}}{p(\omega) T}}\right|^{2} .
$$

[27] The head FTF again can be directly read off equation (14) according to equation (6) and is given by

$$
\Theta_{h}(\omega)=\frac{1}{\omega^{2} S^{2}}\left|1-\frac{\cosh \left[p(\omega)\left(\frac{x}{L}-1\right)\right]}{\frac{p(\omega) T}{\alpha_{c} L^{2}} \sinh [p(\omega)]+\cosh [p(\omega)]}\right|^{2} .
$$

[28] Note that for $\omega \tau_{L} \gg 1$, the head FTF $\Theta_{h}(x, \omega) \sim \alpha_{c}^{-2} \Theta_{q}(\omega) \propto \omega^{-2}$, and both scale as $\omega^{-2}$. The behaviors of the discharge FTF and the heat FTF are illustrated in Figures 2 and 3. As illustrated in Figure 2 for decreasing frequencies $\omega \tau_{L} \ll 1, \Theta_{q}(\omega)$ approaches one. Unlike for the LDD model, here the behavior of the head FTF for $\omega \tau_{L} \gg\left(\alpha_{c} L\right)^{2} / T^{2}$, see Figure 3, is independent from the observation point $x$, as is the case for the LR model [e.g., Gelhar, 1974]. In fact, in Appendix A we show that the LR model can be obtained from the horizontal average of the LDC model. For decreasing frequencies

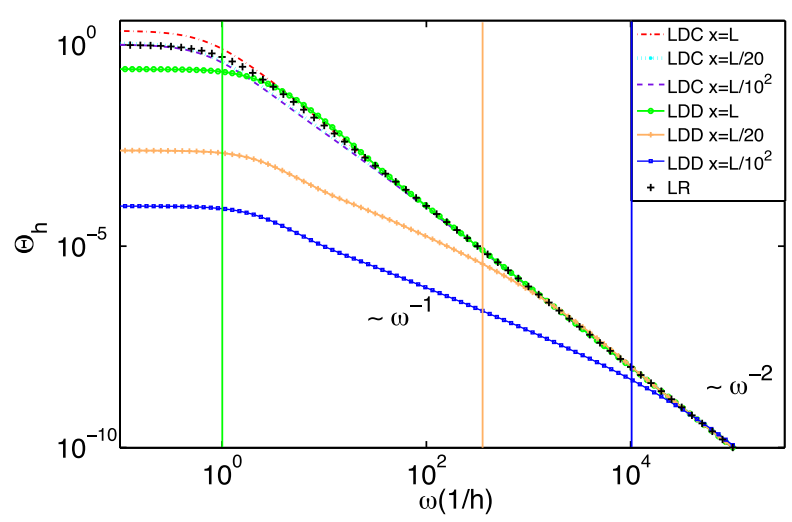

Figure 3. Head FTF $\Theta_{h}(\omega)$ at different observation points for the LR, LDD, and LDC models. The vertical lines indicate the characteristic frequencies $\omega_{x}=T /\left(x^{2} S\right)$ for the observation points (from left to right) $x=L$, $x=L / 20$, and $x=L / 10^{2}$. We used: $S=10^{-4}, T=10^{2} \mathrm{~m}^{2} \mathrm{~h}^{-1}$, $\alpha_{c}=10^{-4} \mathrm{~h}^{-1}$, and $L=10^{3} \mathrm{~m}$. The aquifer response rate is $\omega_{L}=1 \mathrm{~h}$. 

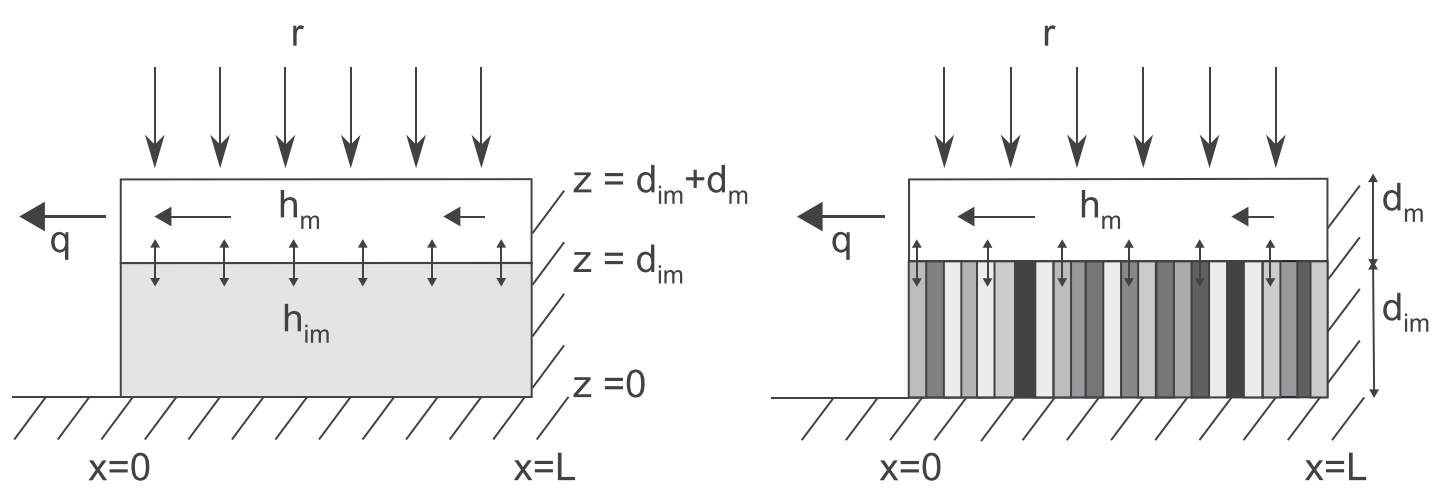

Figure 4. Sketches of the (left) dual continuum and (right) multicontinuum models. The catchment of thickness $d=d_{m}+d_{i m}$ is split into an upper mobile layer of thickness $d_{m}$, and a lower immobile layer of thickness $d_{i m} ; q$ is the stream discharge, $r$ is the aquifer recharge, and $h_{m}$ and $h_{i m}$ are the hydraulic heads in the mobile and in the immobile zones, respectively. The immobile zone of the multicontinuum model is split into slabs of width $\ell \ll L$.

$\omega \tau_{L} \ll 1$, the value of the head FTF at depends on the observation point. Taking the limit $\omega \rightarrow 0$ in the head FTF for the LDC model given in equation (16), we obtain

$$
\lim _{\omega \rightarrow 0} \Theta_{h}(x, \omega)=\left|-\frac{x^{2}}{2 T}+\frac{L x}{T}+\frac{1}{\alpha_{c}}\right|^{2} .
$$

[29] The values of the head FTF decrease at $\omega=0$ as the observation point gets closer to the discharge boundary.

\section{Dual and Multicontinuum Aquifer Models}

[30] In this section, we develop a multicontinuum approach to account for the impact of a spectrum of heterogeneity-induced mass transfer time scales on the aquifer response. The model assumes that the water entering the aquifer infiltrates into a conductive region through which it may flow to the discharge point according to the hydraulic gradient, and from which it may infiltrate into less conductive zones, where it can get trapped and again slowly released. For simplicity, the conductive zone is called mobile region, while the less conductive one is called immobile region. The wide range of temporal scales which characterize natural problems [Tessier et al., 1996; Duffy, 2010], can arise here from a heterogeneous immobile layer.

[31] The aim is to simplify the complex dynamic of aquifer response which occur in a three-dimensional heterogeneous media in a simplified two-dimensional model, which handle heterogeneity in a stochastic framework and returns the range of temporal scales observed in nature. The underlying conceptual models are illustrated schematically in Figure 4. In particular, the simplified conceptual model considers a spatially uniform recharge that reaches the aquifer instantanously, and absence of return flow and overland flow.

[32] We handle spatial heterogeneity in a stochastic framework, where the medium is considered as a particular realization of an ensemble of media with the same statistical properties. The spatially variable hydraulic parameters are modeled as spatial random fields. We consider a stationary and ergodic medium, with a finite correlation length. We assume that the heterogeneity scale is much smaller than the aquifer scale. Thus, we assume that the medium is organized in vertical bins. Constant hydraulic properties are assigned to each bin. The individual values are sampled from independent identically distributed random variables. Thus, such medium can be completely defined by single point distributions of the hydraulic parameters. A schema of the model is given in Figure 4. Figure 4 in the right illustrates the multicontinuum model, where heterogeneity of the immobile layer is organized in bins of width $\ell$ that are far smaller than the aquifer scale. For completeness, we take into account also the case of a homogeneous immobile layer, which reduces the multicontinuum recharge model to a dual continuum recharge model, as illustrated in the left of Figure 4.

\subsection{Model Derivation}

[33] The governing equation of the multicontinuum recharge model is given by the flow equation [De Marsily, 1986]:

$$
s(\mathbf{x}) \frac{\partial h(\mathbf{x}, t)}{\partial t}-\nabla \cdot[K(\mathbf{x}) \nabla h(\mathbf{x}, t)]=0,
$$

where $\mathbf{x}=(x, z)^{T}, s(\mathbf{x})$ is the specific storativity and $K(\mathbf{x})$ is the hydraulic conductivity, see Figure 4. In order to derive the multicontinuum model, we need to resolve explicitly the model taking into account the $z$ direction and the recharge term is considered as upper boundary condition. In the immobile region, $0<z<d_{i m}$, specific storativity and hydraulic conductivity are variable in $x$ direction, $s(\mathbf{x})=s_{i m}$ $(x)$ and $K(\mathbf{x})=K_{i m}(x)$, while in the mobile region, $d_{i m}<z<d$, both specific storativity and hydraulic conductivity are taken constant, $s(\mathbf{x})=s_{m}$ and $K(\mathbf{x})=K_{m}$.

[34] The system is driven by the recharge $r(t)$ at the upper horizontal boundary of the mobile layer, which yields the boundary condition

$$
K_{m} \frac{\partial h(\mathbf{x}, t)}{\partial z}=r(t), \quad z=d .
$$

[35] At the lower boundary a no-flux boundary condition is specified 


$$
K_{i m}(x) \frac{\partial h(\mathbf{x}, t)}{\partial z}=0, \quad z=0 .
$$

[36] As initial condition, we set $h(\mathbf{x}, 0)=0$ in the whole domain. The hydraulic heads in the mobile and immobile regions are denoted by $h_{i m}(\mathbf{x}, t)=h(\mathbf{x}, t)$ for $0<z<d_{i m}$, and $h_{m}(\mathbf{x}, t)=h(\mathbf{x}, t)$ for $d_{i m}<z<d$. Both head and flux are continuous at the interface between the two mobile and immobile regions

$$
h_{m}(\mathbf{x}, t)=h_{i m}(\mathbf{x}, t), \quad K_{m} \frac{\partial h_{m}(\mathbf{x}, t)}{\partial z}=K_{i m}(x) \frac{\partial h_{i m}(\mathbf{x}, t)}{\partial z}, \quad z=d_{i m}
$$

\subsubsection{Vertical Average}

[37] In order to obtain an effective description, we average the flow equation (18) vertically. In the following, we use the same assumption of the classical Dupuit model [e.g., Bear, 1972]. In particular, we neglect geometry variations in response to changes in head so that the flow problem can be linearized [Gelhar and Wilson, 1974].

[38] The average hydraulic heads in the mobile and immobile regions are defined as

$$
\bar{h}_{i m}(x, t)=\frac{1}{d_{i m}} \int_{0}^{d_{i m}} d z h_{i m}(\mathbf{x}, t), \bar{h}_{m}(x, t)=\frac{1}{d_{m}} \int_{d_{i m}}^{d} d z h_{m}(\mathbf{x}, t) .
$$

[39] By integration of equation (18) over the mobile region, we obtain for $\bar{h}_{m}(x, t)$ the governing equation

$$
S_{m} \frac{\partial \bar{h}_{m}(x, t)}{\partial t}-T_{m} \frac{\partial^{2} \bar{h}_{m}(x, t)}{\partial x^{2}}=r(t)-\left.K_{i m}(x) \frac{\partial h_{i m}(\mathbf{x}, t)}{\partial z}\right|_{z=d_{i m}},
$$

where we used the continuity condition (21) and defined the storage capacity $S_{m}=s_{m} d_{m}$ and transmissivity $T_{m}=K_{m}$ $d_{m}$ of the mobile region. The last term in equation (23) represents the flux at the interface between mobile and immobile regions and it is obtained from the solution of the flow equation in the immobile domain, which is given by

$$
s_{i m}(x) \frac{\partial h_{i m}(\mathbf{x}, t)}{\partial t}-K_{i m}(x) \frac{\partial^{2} h_{i m}(\mathbf{x}, t)}{\partial z^{2}}=0 .
$$

[40] Note that we have disregarded flow in horizontal direction in the immobile layer because it is assumed to be small compared to the one in the more conductive region. Furthermore, we assume that vertical equilibrium in the mobile layer is reached fast so that we can set $h_{m}(\mathbf{x}, t) \approx \bar{h}_{m}(x, t)$, which corresponds to the classical Dupuit-Forchheimer approximation [Bear, 1972].

[41] Thus, the boundary condition for $h_{i m}(\mathbf{x}, t)$ at $z=d_{i m}$, which follows from the continuity condition (21), can be written as $h_{i m}(\mathbf{x}, t)=\bar{h}_{m}(x, t)$. At $z=0$, the no-flux condition reads as $\partial h_{i m}(\mathbf{x}, t) / \partial z=0$. The averaged immobile head can be expressed in terms of the convolution product [e.g., Haggerty and Gorelick, 1995; Carrera et al., 1998]

$$
\bar{h}_{i m}(x, t)=\int_{0}^{t} d t^{\prime} g\left[t-t^{\prime}, \tau_{i m}(x)\right] \bar{h}_{m}\left(x, t^{\prime}\right)
$$

where the characteristic time scale

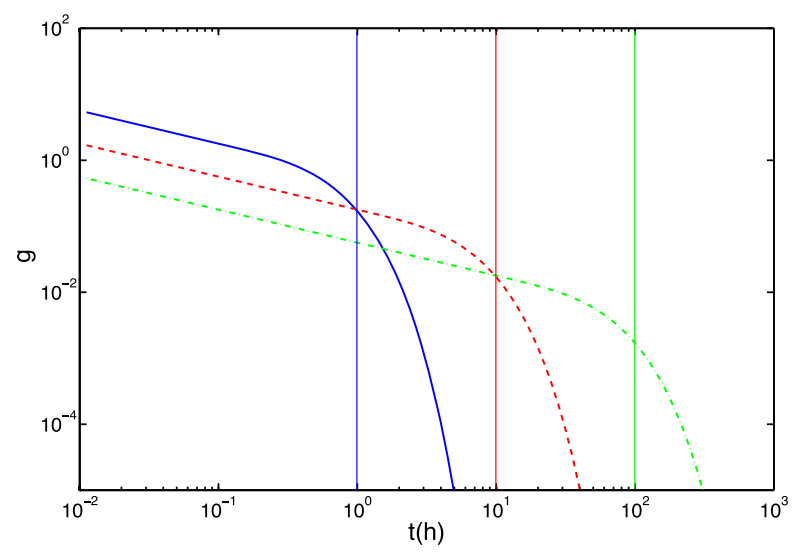

Figure 5. Temporal evolution of $g\left(t, \tau_{i m}\right)$ for (blue solid) $\tau_{i m}=1 \mathrm{~h}$, (red dashed) $\tau_{i m}=10 \mathrm{~h}$ and (green dash-dotted) $\tau_{\text {im }}=10^{2} \mathrm{~h}$.

$$
\tau_{i m}(x)=\frac{d_{i m}^{2} s_{i m}(x)}{K_{i m}(x)}
$$

denotes the typical mean time for the immobile zone to equilibrate with the mobile zone. The kernel function $g\left[t, \tau_{i m}(x)\right]$ is obtained from the solution of equation (24) for the boundary condition $h_{i m}(\mathbf{x}, t)=\delta(t)$ at $z=d_{i m}$. In Fourier space, it is given by

$$
\tilde{g}\left[\omega, \tau_{i m}(x)\right]=\frac{1}{\sqrt{i \omega \tau_{i m}(x)}} \tanh \left[\sqrt{i \omega \tau_{i m}(x)}\right] .
$$

[42] The temporal behavior of $g\left[t, \tau_{i m}(x)\right]$, see Figure 5, is well known from multirate mass transfer models for solute transport in multicontinuum media [Haggerty and Gorelick, 1995; Carrera et al., 1998]. For $t \ll \tau_{\text {im }}(x)$ one observes the characteristic $t^{-1 / 2}$ behavior, and for $t \gg \tau_{\text {im }}(x)$ an exponential break-off.

[43] By vertical integration of equation (24), we find that the flux term on the right side of equation (23) is equal to the time derivative of equation (25). Using this result in equation (23), we obtain the nonlocal flow equation

$$
S_{m} \frac{\partial \bar{h}_{m}(x, t)}{\partial t}+\frac{\partial}{\partial t} \int_{0}^{t} d t^{\prime} \varphi\left(x, t-t^{\prime}\right) \bar{h}_{m}\left(x, t^{\prime}\right)-T_{m} \frac{\partial^{2} \bar{h}_{m}(x, t)}{\partial^{2} x}=r(t),
$$

where we define the memory function $\varphi(x, t)$ as

$$
\varphi(x, t)=S_{i m}(x) g\left[t, \tau_{i m}(x)\right]
$$

with $S_{i m}(x)=d_{i m} s_{i m}(x)$. Note that equation (28) is similar to the LD model given in equation (1), but reflects the presence of different storage time scales in the medium, which gives rise to the nonlocal term. The memory function represents the dynamics of the recharge and storage process in the immobile layer. In this sense, equation (28) can be seen as a nonlocal in time LD model.

\subsubsection{Ensemble Average}

[44] We handle spatial heterogeneity of the immobile zone in a stochastic framework that models the sequence of hydraulic parameters in the immobile region illustrated 
in Figure 4 as a spatial random process. Hydraulic parameters are assigned independently to the slabs and thus the medium is completely defined by the single point distribution of immobile conductivities. Note that the spatial process of the hydraulic parameters is stationary and ergodic by definition, while the head related process is not stationary, as it varies as a function of the location.

[45] In order to obtain an upscaled effective formulation, we average equation (28) over the ensemble of immobile conductivities. This gives

$S_{m} \frac{\partial\left\langle\bar{h}_{m}(x, t)\right\rangle}{\partial t}+\frac{\partial}{\partial t} \int_{0}^{t} d t^{\prime}\left\langle\varphi\left(t-t^{\prime} \mid x\right) h_{m}\left(x, t^{\prime}\right)\right\rangle-T_{m} \frac{\partial^{2}\left\langle\bar{h}_{m}(x, t)\right\rangle}{\partial x^{2}}=r(t)$

where the angular brackets indicate ensemble average. For the ensemble average of the second term on the left side of equation (30), we use the mean field approximation

$$
\left\langle\varphi\left(t-t^{\prime} \mid x\right) \bar{h}_{m}\left(x, t^{\prime}\right)\right\rangle \approx\left\langle\varphi\left(t-t^{\prime} \mid x\right)\right\rangle\left\langle\bar{h}_{m}\left(x, t^{\prime}\right)\right\rangle,
$$

which is justified when the spatial extension of $\bar{h}_{m}(x, t)$ is much larger than the width $\ell$ of a slab, see Figure 4 . Under this condition, the head has sampled a representative part of the medium heterogeneity. Considering that the typical diffusion time for the hydraulic head in the mobile layer over the heterogeneity scale is $S_{m} \ell^{2} / T_{m}$, we can assume that for times larger that this diffusion scale, enough heterogeneity has been sampled and the mean field approximation is valid. A more rigorous analysis of the validity of this approximation and a quantification of the sample to sample fluctuations is left for future work. Note that in similar multirate mass transfer (MRMT) models for solute transport in multicontinuum media this approximation is made on a routine basis [Haggerty and Gorelick, 1995; Carrera et al., 1998].

[46] We define the global memory function $\varphi(t)$ by the ensemble average of the local memory functions $\varphi(t \mid x)$ given by equation (29),

$$
\varphi(t)=\langle\varphi(t \mid x)\rangle .
$$

[47] Details on the ensemble average are given in Appendix B. Thus, we obtain the following upscaled governing equation:

$S_{m} \frac{\partial\left\langle\bar{h}_{m}(x, t)\right\rangle}{\partial t}+\frac{\partial}{\partial t} \int_{0}^{t} d t^{\prime} \varphi\left(t-t^{\prime}\right)\left\langle\bar{h}_{m}\left(x, t^{\prime}\right)\right\rangle-T_{m} \frac{\partial^{2}\left\langle\bar{h}_{m}(x, t)\right\rangle}{\partial^{2} x}=r(t)$.

[48] Note that when the mobile and immobile zones are vertically in equilibrium, equation (33) reduces to a local flow equation for an equivalent homogeneous medium

$$
S_{e} \frac{\partial\left\langle\bar{h}_{m}(x, t)\right\rangle}{\partial t}-T_{m} \frac{\partial^{2}\left\langle\bar{h}_{m}(x, t)\right\rangle}{\partial^{2} x}=r(t)
$$

with the total immobile storativity given by

$$
S_{e}=S_{m}+\left\langle S_{i m}\right\rangle
$$

where $\left\langle S_{i m}\right\rangle$ is the ensemble average of $S_{i m}(x)$.
[49] Equation (33) can be conveniently solved in frequency domain. Fourier transform of equation (33) gives

$$
i \omega\left[S_{m}+\tilde{\varphi}(\omega)\right]\left\langle\tilde{h}_{m}(x, \omega)\right\rangle-T_{m} \frac{\partial^{2}\left\langle\tilde{h}_{m}(x, \omega)\right\rangle}{\partial^{2} x}=\tilde{r}(\omega),
$$

[50] In frequency domain, the governing equation of the multicontinuum recharge model (36) is formally identical to the Fourier transform of the LD model given in equation (1). Consequently, the expression for the FTF for the recharge multicontinuum models, can be obtained by substituting $S \rightarrow S_{m}+\tilde{\varphi}(\omega)$ in the respective expressions for the LD models. Solutions for the multicontinuum models with Dirichlet and Cauchy boundary conditions at the outlet are given in Appendix C.

\subsection{Dual Continuum}

[51] For a homogeneous immobile zone, we obtain the dual continuum recharge model by setting $S_{i m}(x)=S_{i m}=$ const . and $K_{i m}(x)=K_{i m}=$ const . in equations (28) and (29). In the dual continuum model, both mobile and storage layer are characterized by uniform but clearly distinct hydraulic properties. We distinguish here the following relevant time scales, which determine the behavior of the catchment response: the time scale for head propagation along the full catchment in the mobile zone, $\tau_{M}$, the time scale $\tau_{E}$ for the head propagation along the catchment in the asymptotic equilibrium model (34), and the relaxation time scale $\tau_{i m}$ to reach equilibrium between mobile and immobile zones,

$$
\tau_{M}=\frac{S_{m} L^{2}}{T_{m}}, \quad \tau_{E}=\frac{S_{e} L^{2}}{T_{m}} \quad \tau_{i m}=\frac{s_{i m} d_{i m}^{2}}{K_{i m}} .
$$

[52] Furthermore, we identify the time scale $\tau_{a}$ that sets the time for activating the immobile zone. For time larger than $\tau_{a}$ enough water enters the immobile zone and the contribution of the immobile zone is relevant in the dynamic of the whole system. The immobile zone is activated if the proportion of immobile storativity accessed at time $\tau_{a}$ is comparable to the mobile storativity, that is, when $S_{i m} \sqrt{\tau_{a} / \tau_{\text {im }}}=S_{m}$, which implies

$$
\tau_{a}=\tau_{i m}\left(\frac{S_{m}}{S_{i m}}\right)^{2}
$$

and this means it depends on the ratio of mobile and immobile storativities. In order to observe the impact of the immobile zone on the aquifer dynamics, the activation and relaxation time scales need to be clearly separated, $\tau_{a} \ll \tau_{\text {im }}$, which implies $\left(S_{m} / S_{i m}\right)^{2} \ll 1$. In the following, we study the frequency response of the dual continuum aquifer model for Dirichlet and Cauchy boundary conditions at the outlet.

[53] The behavior of the discharge frequency transfer function $\Theta_{q}(\omega)$ at the outlet is shown in Figure 6. We read the figure from right to left, which corresponds to early and late times. For $\omega>\tau_{a}^{-1}$, i.e., before the immobile storage is activated, flow takes place primarily in the mobile zone and $\Theta_{q}(\omega)$ behaves as in a homogeneous medium characterized by the parameters of the mobile zone only. For decreasing frequencies, $\omega \ll \tau_{a}^{-1}$, the immobile zone is activated. In 


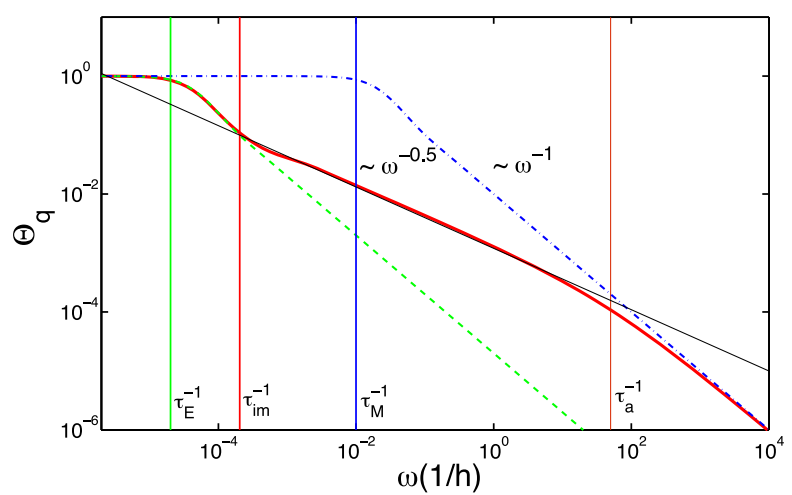

Figure 6. Discharge FTF $\Theta_{q}(\omega)$ for the dual continuum model with Dirichlet BC (red solid) given by (C3) with the memory function (29). (Blue dash-dotted) $\Theta_{q}(\omega)$ for the homogeneous LDD model characterized by $S_{m}$ and $T_{m}$. (Green dashed) $\Theta_{q}(\omega)$ for the equivalent homogeneous LDD model (34) characterized by the effective storativity $S_{e}=S_{m}+S_{i m}$ and $T_{m}$. We used $S_{m}=10^{-4} \mathrm{~m}^{-1}, S_{i m}=5 \times 10^{-2}$ $\mathrm{m}^{-1}, T_{m}=1 \mathrm{mh}^{-1}, T_{i m}=10^{-1} \mathrm{mh}^{-1}, L=10^{3} \mathrm{~m}$. The time scales are $\tau_{a}=0.02 \mathrm{~h}, \tau_{M}=10^{2} \mathrm{~h}, \tau_{i m}=5 \times 10^{3} \mathrm{~h}, \tau_{E}=5.01 \times$ $10^{4} \mathrm{~h}$. The solid black line indicates $\omega^{-1 / 2}$.

the frequency regime $\tau_{i m}^{-1} \ll \omega \ll \tau_{a}^{-1}, \quad \Theta_{q}(\omega)$ scales anomalously as $\omega^{-1 / 2}$, see Appendix D1, while the homogeneous LDD model scales as $\Theta_{q}(\omega) \propto \omega^{-1}$. For frequencies $\omega<\tau_{i m}^{-1}$, or equivalently times larger than the relaxation time scale $\tau_{i m}$, the system behaves as an equivalent homogeneous Dupuit aquifer characterized by the effective storativity $S_{e}=S_{m}+S_{i m}$, see equation (34).

[54] As discussed previously for the homogeneous LDD model, also here the scaling of the head FTF depends on the position in the aquifer. Close to the outfall at $x \ll L$, the head FTF scales as $\Theta_{h}(\omega) \sim \omega^{-1 / 2}$ in the frequency regime $\tau_{i m}^{-1} \ll \omega \ll \tau_{a}^{-1}$, when the immobile zone is activated, while the homogeneous aquifer scales as $\omega^{-1}$. For $\omega>\tau_{a}^{-1}$, it scales as the homogeneous LDD model, see Appendix $\mathrm{C} 1$ for an analytical solution of the head FTF and Appendix D1 for the scalings. For conciseness, here and in the following we do not show the corresponding figures of the scaling of the head FTF.

[55] Figure 7 shows the discharge FTF $\Theta_{q}(\omega)$ for the dual continuum aquifer model with Cauchy boundary conditions. For frequencies $\omega \gg \tau_{a}^{-1}$, that is, before the activation of the immobile zone, $\Theta_{q}(\omega)$ shows the same behavior as a homogeneous LDC model characterized by the parameters $S_{m}$ and $T_{m}$ of the mobile zone only. In the regime $\tau_{\text {im }}^{-1} \ll \omega \ll \tau_{a}^{-1}$, the frequency response shows the scaling $\omega^{-1}$, while the homogeneous LDC models predicts $\Theta_{q}(\omega) \propto \omega^{-2}$ for $\omega \gg \tau_{M}$. Finally, for $\omega<\tau_{i m}^{-1}$, the frequency response behaves as the equivalent homogeneous LDC model given by equation (34) with Cauchy boundary conditions.

[56] Thus, note that a discharge behavior of $\Theta_{q}(\omega)$ $\propto \omega^{-1}$ does not necessarily mean that the catchment dynamics can be described by a homogeneous LDD aquifer. The catchment may actually rather be a LDC aquifer with a large storage capacity in the immobile zone.

[57] As for the homogeneous LDC model, the head FTF scales as the discharge FTF, $\Theta_{h}(\omega) \sim \Theta_{q}(\omega)$, see Appendix C2 for analytical solution and Appendix D1.

\subsection{Multicontinuum}

[58] Here we consider a multicontinuum recharge model characterized by an heterogeneous immobile zone that wants to relate the wide range of relaxation time scales observed in nature to a stochastic description of the medium heterogeneity, as discussed in section 3.1.2. Hydraulic conductivity can vary over many orders of magnitude even in apparently homogeneous media, while specific storativity is typically much less variable [Warren and Root, 1963]. Thus, we consider in the following the scenario of constant $s_{i m}(x)=s_{i m}$ and variable $K_{i m}(x)$, see Appendix B. A broad distribution of hydraulic conductivity values renders a wide range of temporal scales. The distribution of immobile times $\tau$ is expressed in terms of the conductivity distribution $P_{k}(k)$ as

$$
P_{i m}(\tau)=\frac{d_{i m}^{2} s_{i m}}{\tau^{2}} P_{k}\left(\frac{d_{i m}^{2} s_{i m}}{\tau}\right),
$$

see Appendix B.

[59] The memory function $\varphi(t)$, equation (32), then is given by the ensemble average of the local memory functions associated to each immobile zone $g(t, \tau)$ as

$$
\varphi(t)=S_{i m} \int_{0}^{\infty} d \tau P_{i m}(\tau) g(t, \tau),
$$

where $g(t, \tau)$ is defined in terms of its Fourier transform (27). In order to account for a broad distribution of mass transfer scales we employ a truncated power-law distribution for the characteristic times in the immobile zones

$$
P_{\text {im }}(\tau)=\frac{1-\beta}{\tau_{2}\left(1-\tau_{1} / \tau_{2}\right)^{1-\beta}}\left(\frac{\tau}{\tau_{2}}\right)^{-\beta} \mathcal{H}\left(\tau-\tau_{2}\right) \mathcal{H}\left(\tau_{1}-\tau\right)
$$

with $\mathcal{H}(\tau)$ the Heaviside step function, $\tau_{1}$ and $\tau_{2}$, respectively, the smallest and the largest cut offs of the truncated

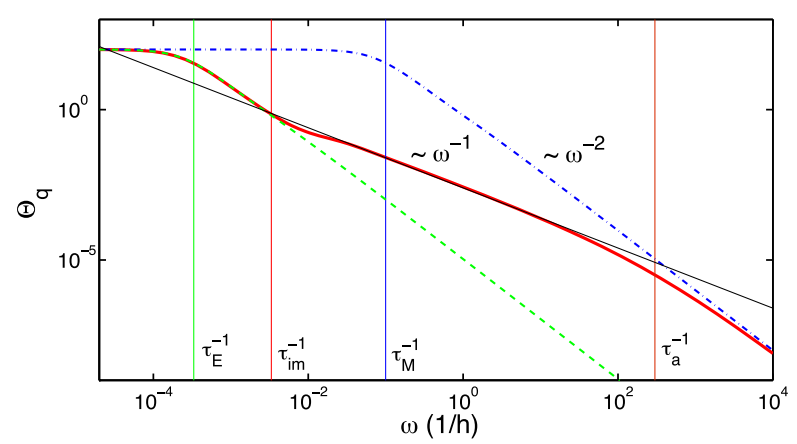

Figure 7. (Red solid) Discharge FTF $\Theta_{q}(\omega)$ for the dual continuum model with Cauchy $\mathrm{BC}$ given by (C6) with the memory function (29). (Blue dash-dotted) $\Theta_{q}(\omega)$ for the homogeneous LDC model characterized by the parameters $S_{m}$ and $T_{m}$ of the mobile zone. (Green dashed) $\Theta_{q}(\omega)$ for the equivalent homogeneous LDC model (34) characterized by the effective storativity $S_{e}=S_{m}+S_{i m}$ and $T_{m}$. We used $S_{m}=$ $10^{-4} \mathrm{~m}^{-1}, S_{i m}=3 \times 10^{-4} \mathrm{~m}^{-1}, T_{m}=10 \mathrm{mh}^{-1}, T_{i m}=1 \mathrm{mh}^{-1}$, $L=10^{3} \mathrm{~m}, \quad \alpha_{c}=10^{-4} \mathrm{~h}^{-1}$. The time scales are $\tau_{a}=3.3 \times$ $10^{-3} \mathrm{~h}, t_{M}=10 \mathrm{~h}, \tau_{\text {im }}=300 \mathrm{~h}, \tau_{E}=3010 \mathrm{~h} \simeq 125 \mathrm{~d}$. The solid black line indicates $\omega^{-1}$. 


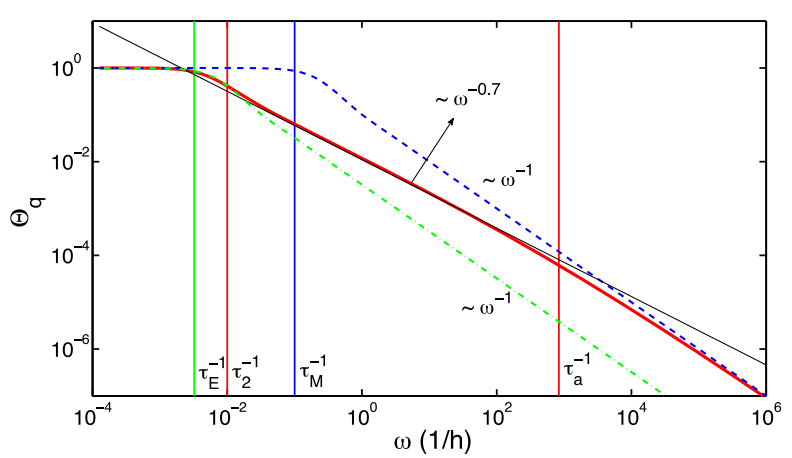

Figure 8. (Red solid) Discharge FTF $\Theta_{q}(\omega)$ for the multicontinuum model with Dirichlet $\mathrm{BC}$ given by $(\mathrm{C} 3)$ with the memory function (42). (Blue dash-dotted) $\Theta_{q}(\omega)$ for the homogeneous LDD model characterized by the parameters $S_{m}$ and $T_{m}$ of the mobile zone. (Green dashed) $\Theta_{q}(\omega)$ for the equivalent homogeneous LDD model (34) characterized by the effective storativity $S_{e}=S_{m}+S_{i m}$ and $T_{m}$. We used $S_{m}=10^{-3} \mathrm{~m}^{-1}, S_{i m}=3 \times 10^{-2} \mathrm{~m}^{-1}, T_{m}=10 \mathrm{mh}^{-1}, L=$ $10^{3} \mathrm{~m}, \beta=0.7$. The time scales are $\tau_{E}=310 \mathrm{~h}, \tau_{2}=10^{2} \mathrm{~h}$, $\tau_{M}=10 \mathrm{~h}$, and $\tau_{a}=1.2 \times 10^{-3} \mathrm{~h}$.

power-law distribution of $\tau$ and $1 / 2<\beta<1$. The time scales $\tau_{1}$ and $\tau_{2}$ are related to the largest and smallest conductivity values in the immobile region, respectively. Specifically $\tau_{1}$ is restricted by the hypothesis $K_{m} \ll K_{i m}(x)$. The timescale $\tau_{2}$, which is the largest storage time for the multicontinuum model, corresponds to the timescale $\tau_{i m}$ given in equation (26) for the double-continuum model.

[60] The memory function $\varphi(t)$ for the distribution (41) is approximated by the truncated power-law distribution

$$
\varphi(t)=\frac{S_{i m}}{\tau_{2} \Gamma(1-\beta)}\left(\frac{\tau}{\tau_{2}}\right)^{-\beta} \exp \left(-\frac{\tau}{\tau_{2}}\right),
$$

see Appendix D. The Fourier transform of equation (42) is given by

$$
\tilde{\varphi}(\omega)=S_{\text {im }}\left(i \omega \tau_{2}+1\right)^{\beta-1} .
$$

[61] The time scale for the activation of the immobile zones $\tau_{a}$ here is given by

$$
\tau_{a}=\tau_{2}\left(\frac{S_{m}}{S_{i m}}\right)^{\frac{1}{1-\beta}}
$$

[62] It denotes the time for which the proportion of active immobile storativity is equal to the mobile storativity, $S_{i m}\left(\tau_{a} / \tau_{2}\right)^{1-\beta}=S_{m}$. In the following, we study the frequency response of the multicontinuum aquifer model for both Dirichlet and Cauchy boundary conditions at the outfall. Figure 8 shows the discharge FTF in the multicontinuum aquifer model for Dirichlet boundary conditions. The storage time distribution (41) is characterized by $\beta=0.7$. We clearly distinguish three different recharge regimes that are marked by the time scales $\tau_{M}$ for head propagation in the mobile zone, $\tau_{E}$, the time scale for head propagation in the effective asymptotic LDD aquifer and the activation time scale $\tau_{a}$, equation (44), for the immobile zones. As before, we read the figure from right to left. At frequencies larger than $\tau_{a}^{-1}$, that is, at time smaller than the activation scale $\tau_{a}$, the discharge FTF behaves as the corresponding LDD model characterized by the mobile zone parameters $S_{m}$ and $T_{m}$ because flow is predominantly taking place in the mobile region. In the intermediate regime $\tau_{2}^{-1} \ll \omega \ll \tau_{a}^{-1}$, the storage layer is activated the discharge FTF behaves anomalously. Here it scales as $\omega^{-0.7}$. As shown in Appendix D2, $\Theta_{q}(\omega)$ in this regime behaves according to

$$
\Theta_{q}(\omega) \propto \omega^{-\beta},
$$

while the homogeneous LDD aquifer scales as $\omega^{-1}$. Asymptotically, at the frequency $\omega \ll \tau_{2}^{-1}$ the mobile layer is in equilibrium with the immobile storage region and the system behaves as an equivalent homogeneous LD aquifer given in equation (34). In this frequency regime, the discharge FTF crosses over to the small frequency plateau, which it assumes on the scale $\tau_{E}^{-1}$.

[63] The evolution of the head FTF depends on the position $x$ in the aquifer as for the homogeneous LDD model. Near the discharge point at $x \ll L$, it scales as $\Theta_{h}(\omega)$ $\sim \omega^{-\beta}$ for $\tau_{2}^{-1} \ll \omega \ll \tau_{a}^{-1}$, the regime of activation of the heterogeneous immobile zone. For $\omega>\tau_{a}^{-1}$, it scales as the LDD model, see also Appendix C1 and Appendix D2.

[64] The behavior of the multicontinuum aquifer for Cauchy boundary conditions, shown in Figure 9 for $\beta=0.7$ is qualitatively similar. However, in the frequency regime $\tau_{2}^{-1} \ll \omega \ll \tau_{a}^{-1}$ the discharge FTF scales as

$$
\Theta_{q}(\omega) \propto \omega^{-2 \beta}
$$

while the homogeneous LDC aquifer scales as $\omega^{-2}$. As for the homogeneous LDC model, the head FTF scales as the discharge FTF $\Theta_{h}(x, \omega) \sim \Theta_{q}(\omega)$, see also Appendix C2 and Appendix D2.

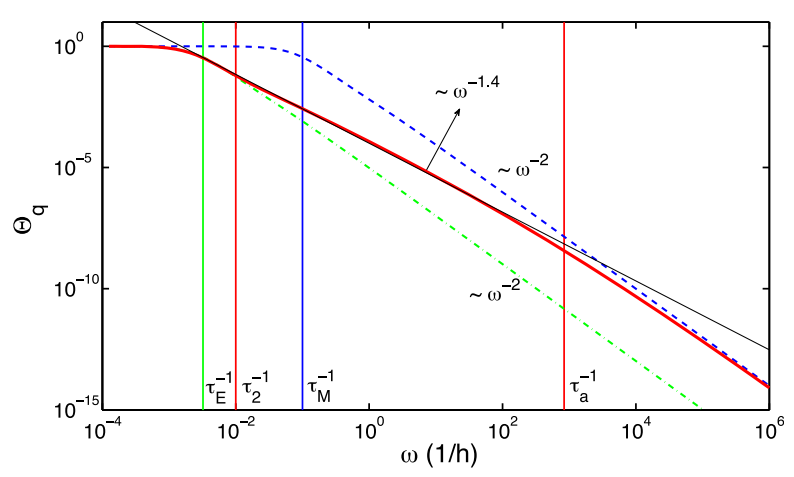

Figure 9. (Red solid) Discharge FTF $\Theta_{q}(\omega)$ for the multicontinuum model with Cauchy BC given by (C6) with the memory function (42). (Blue dash-dotted) $\Theta_{q}(\omega)$ for the homogeneous LDC model characterized by the parameters $S_{m}$ and $T_{m}$ of the mobile zone. (Green dashed) $\Theta_{q}(\omega)$ for the equivalent homogeneous LDC model (34) characterized by the effective storativity $S_{e}=S_{m}+S_{i m}$ and $T_{m}$. We used $S_{m}$ $=10^{-3} \mathrm{~m}^{-1}, \quad S_{i m}=3 \times 10^{-2} \mathrm{~m}^{-1}, \quad T_{m}=10 \mathrm{mh}^{-1}, \quad L=10^{3} \mathrm{~m}$, $\alpha_{c}=10^{-4} \mathrm{~h}^{-1}, \beta=0.7$. The time scales are $\tau_{E}=310 \mathrm{~h}$, $\tau_{2}=10^{2} \mathrm{~h}, \tau_{M}=10 \mathrm{~h}$, and $\tau_{a}=1.2 \times 10^{-3} \mathrm{~h}$. 


\section{Conclusions}

[65] In this paper, we present a relatively simple multicontinuum approach that may be used to link the scaling of the discharge FTF to a stochastic description of the catchment heterogeneity. Specifically, the proposed model allows to relate the scaling of the FTF to physical parameters as storativity, the catchment size and its discharge boundary condition, on one hand, and to a stochastic description of heterogeneous hydraulic conductivity, on the other. In particular, we emphasize the role played by the discharge BC in the scaling of the FTF. Specifically, the scaling of the discharge FTF $\Theta_{q}(\omega) \propto \omega^{-\beta}$ with $1 / 2<\beta$ $<1$ may be explained by a LD aquifer model with Dirichlet boundary conditions at the outfall, while exponents 1 $<\beta<2$ may be attributed to a LD model with Cauchy boundary condition and a large heterogeneous immobile storage capacity. We hypothesize that by modeling the FTF of an aquifer using the proposed approach, it may be possible to extract information on the physical aquifer properties that can be crossed-checked by a geostatistical characterization of the catchment heterogeneity.

[66] The proposed model may be used to study the discharge and head dynamics of small catchments at hillslope scale so that the recharge can be assumed uniform. The model may be particularly suitable for fractured aquifers, where the conductive fractures can be considered as the "mobile" zone and the interfacture matrix as the "immobile" zones.

[67] The dual and multicontinuum approaches provide an alternative to existing fractal aquifer models for the interpretation of observed fractal discharge characteristics, and allow to relate these behaviors to a stochastic description of aquifer heterogeneity as reflected in the distribution of characteristic storage time scales.

\section{Appendix A: Linear Reservoir Model}

[68] The LR model is a zero-dimensional model that disregards spatial variations of the water level and the hydraulic head $h(t)$ is a function of time only [Gelhar, 1974; Bear, 1972], see Figure 1. The governing equation is

$$
S \frac{d h(t)}{d t}=-q(t)+r(t), \quad q(t)=\alpha\left[h(t)-h_{0}\right],
$$

where $\alpha$ is an outflow constant $\left(\mathrm{T}^{-1}\right)$. The discharge FTF is

$$
\Theta_{q}(\omega)=\frac{1}{1+\left(\tau_{\alpha} \omega\right)^{2}},
$$

where $\tau_{\alpha}$ is a characteristic response time given by $\tau_{\alpha}=S / \alpha$ [Gelhar and Wilson, 1974]. Frequencies lower than $\tau_{\alpha}^{-1}$ are not damped by the system, while for higher frequencies the transfer function scales as $\Theta_{q}(\omega) \sim \omega^{-2}$. The head FTF reads as [see also e.g., Gelhar, 1974]

$$
\Theta_{h}(\omega)=\frac{1}{\alpha^{2}+(\omega S)^{2}}
$$

[69] The scaling for large frequencies for both the groundwater discharge and the groundwater level is the same $\Theta_{h}(\omega) \sim \Theta_{q}(\omega) \sim \omega^{-2}$.
[70] Note that Gelhar [1974] derived the outflow constant $\alpha$, which is characteristic of the LR model, from the LDD model and obtained $\alpha=L^{2} /(3 T)$. As outlined above, the behaviors of the LDC and LDD models are different. Thus, we propose to determine $\alpha$ by comparing the response times $\tau_{L}$ of the LR and LDC models which are $\tau_{L}=L^{2} S / T$ and $\tau_{\alpha}=S / \alpha$, respectively. This leads to $\alpha=T / L^{2}$.

[71] We derive now the zero-dimensional LR model from a higher dimensional model. The horizontal average over the catchment is defined as

$$
\langle h(t)\rangle_{h}=\frac{1}{L} \int_{0}^{L} h(x, t) d x
$$

where the subscript $h$ here denotes horizontal. Averaging of equation (1) using the Cauchy boundary conditions (13), we obtain

$$
S \frac{d\langle h(t)\rangle_{h}}{d t}=-\alpha\left[h(0, t)-h_{0}\right]+r(t) .
$$

[72] Expression (A5) is analogous to the governing equation of the LR model given in equation (A(1)), except for the local term of $h(0, t)$ at the discharge boundary. The two equations are equivalent if one can set $h(0, t) \simeq\langle h(t)\rangle$, which implies that the piezometric gradient in the aquifer is small. Furthermore, without any assumption on the piezometric gradient, the two models are equivalent at very small or large times. At large times, for $t \gg \tau_{L}$ with $\tau_{L}=L^{2} S / T$ the system is horizontally in equilibrium, $h(x, t)=\langle h(t)\rangle_{h}$ and the two models are equivalent. At short times $t \ll \tau_{L}$, the LR model and the integrated LD model with Cauchy BC coincide for the equilibrium initial conditions $h(x, 0)=h_{0}$. This implies that for $t \ll \tau_{L}, h(0, t) \simeq\langle h(t)\rangle_{h}$. Indeed horizontal integration of the head FTF for the LDC model (16), gives

$$
\left\langle\Theta_{h}(\omega)\right\rangle_{h}=\frac{1}{L} \int_{0}^{L} \Theta_{h}(x, \omega) d x=\left|\frac{1}{i \omega S}\left\{1-\frac{\alpha}{i \omega S} \frac{\tanh [p(\omega)]}{\tanh [p(\omega)]+\frac{\alpha L^{2}}{T p(\omega)}}\right\}\right|^{2}
$$

[73] In the limit of $\omega \ll T /\left(S L^{2}\right)$, first-order Taylor expansion of equation (A6) for $p(\omega) \ll 1$ leads to

$$
\left\langle\Theta_{h}(\omega)\right\rangle_{h}=\left|\frac{1}{\alpha+i \omega S}\right|^{2},
$$

which is equivalent to the head FTF for the LR model given by equation (A3). In fact the characteristic time $\tau_{L}$ denotes the diffusion time over the catchment size $L$. For $t \gg \tau_{L}$, the catchment is horizontally in equilibrium and we can neglect spatial variation of the hydraulic head as in the LR model.

\section{Appendix B:Multicontinuum Model: Distribution of Hydraulic Parameters}

[74] The ensemble average of equation (32) can be executed explicitly. Using expression (29) for $\varphi(x, t)$ and setting $S_{i m}(x)=d_{i m} s_{i m}(x)$, we obtain 


$$
\begin{aligned}
\varphi(t) & =\left\langle s_{i m}(x) d_{i m} g\left[t, \tau_{i m}(x)\right]\right\rangle \\
& =\int_{0}^{\infty} d s \int_{0}^{\infty} d \tau\left\langle\delta\left[s-s_{i m}(x)\right] \delta\left[\tau-\tau_{i m}(x)\right]\right\rangle s d_{i m} g(t, \tau) .
\end{aligned}
$$

[75] This can be written as

$$
\varphi(t)=\int d s \int d \tau P_{s, \tau}(s, \tau) s d_{i m} g(t \mid \tau)
$$

with the joint distribution of $s_{i m}$ and $\tau_{i m}$

$$
P_{s, \tau}(s, \tau)=\left\langle\delta\left[s-s_{i m}(x)\right] \delta\left[\tau-\tau_{i m}(x)\right]\right\rangle .
$$

[76] It can be expressed as

$$
P_{s, \tau}(s, \tau)=P_{\tau}(\tau \mid s) P_{s}(s),
$$

where the conditional distribution $P_{\tau}(\tau \mid s)$ is given in terms of the distribution $P_{k}(k)$ of random conductivities $K_{i m}$ as

$$
P_{\tau}(\tau \mid s)=\frac{d_{i m}^{2} s}{\tau^{2}} P_{k}\left(\frac{d_{i m}^{2} s}{\tau}\right) .
$$

where we used relation (26) between the immobile time $\tau_{i m}$ $(x)$ and conductivity $K_{i m}(x)$ for the mapping $K_{i m}(x)$ on $\tau_{\text {im }}(x)$.

[77] From the definition of $\tilde{g}(\omega, \tau)$ given in equation (27), we note that $\tilde{g}(\omega, \tau)$ is precisely a function of the product $\omega \tau$. This implies that $g(t, \tau)$ can be expressed

$$
g(t, \tau)=\frac{1}{\tau} g^{\prime}\left(\frac{t}{\tau}\right)
$$

where $g^{\prime}(t)=g(t, 1)$. Thus, equation $(\mathrm{B}(1))$ can be written as

$$
\varphi(t)=\int d s \int d \tau P_{\tau}(\tau \mid s) P_{s}(s) \frac{s d_{i m}}{\tau} g^{\prime}\left(\frac{t}{\tau}\right)
$$

[78] As specific storativity is typically much less variable than conductivity, here we set

$$
P_{s}(s)=\delta\left(s-s_{i m}\right)
$$

such that

$$
\varphi(t)=\int d \tau P_{i m}(\tau) \frac{d_{i m} s_{i m}}{\tau} g^{\prime}\left(\frac{t}{\tau}\right)
$$

where define $P_{i m}(\tau)=P_{\tau}\left(\tau \mid s_{i m}\right)$.

\section{Appendix C: Multicontinuum Model: Solutions for the Hydraulic Head}

[79] For completeness, in the following, we give the closed-form solutions for the Fourier transform of the mobile hydraulic head, the head FTF and discharge FTF for both Dirichlet and Cauchy boundary conditions.

\section{C1. Dirichlet Boundary Conditions}

[80] The solution of equation (36) for Dirichlet BC is given by

$$
\left\langle\tilde{h}_{m}(x, \omega)\right\rangle=\frac{R(\omega)}{i \omega\left[S_{m}+\tilde{\varphi}(\omega)\right]}\left\{1-\frac{\cosh \left[p_{e}(\omega)\left(1-\frac{x}{L}\right)\right]}{\cosh \left[p_{e}(\omega)\right]}\right\}
$$

where we defined $p_{e}(\omega)=\sqrt{i \omega \tau_{\text {eff }}(\omega)}$ and $\tau_{\text {eff }}(\omega)=$ $L^{2}\left[S_{m}+\tilde{\varphi}(\omega)\right] / T_{m}$. The head FTF can be directly obtained from equation (C1) according to equation (6) and is given by

$$
\Theta_{h}(x, \omega)=\frac{1}{\omega^{2}\left|S_{m}+\tilde{\varphi}(\omega)\right|^{2}}\left|\left\{1-\frac{\cosh \left[p_{e}(\omega)\left(1-\frac{x}{L}\right)\right]}{\cosh \left[p_{e}(\omega)\right]}\right\}\right|^{2}
$$

[81] The discharge FTF defined in equation (5) is obtained from equations (C(1)) through equation (2) and it reads

$$
\Theta_{q}(\omega)=\frac{S_{m}}{\omega \tau_{M}\left|S_{m}+\tilde{\varphi}(\omega)\right|}\left|\tanh \left[p_{e}(\omega)\right]\right|^{2}
$$

\section{C2. Cauchy Boundary Condition}

[82] For Cauchy BC at the outfall, the solution of equation (36) reads as

$$
\left\langle\tilde{h}_{m}(x, \omega)\right\rangle=\frac{R(\omega)}{i \omega\left[S_{m}+\tilde{\varphi}(\omega)\right]}\left\{1-\frac{\cosh \left[p_{e}(\omega)\left(1-\frac{x}{L}\right)\right]}{\frac{p_{e}(\omega) T_{m}}{\alpha L^{2}} \sinh \left[p_{e}(\omega)\right]+\cosh \left[p_{e}(\omega)\right]}\right\} .
$$

[83] The related head FTF is

$\Theta_{h}(x, \omega)=\frac{1}{\omega^{2}\left|S_{m}+\tilde{\varphi}(\omega)\right|^{2}}\left|1-\frac{\cosh \left[p_{e}(\omega)\left(1-\frac{x}{L}\right)\right]}{\frac{p_{e}(\omega) T_{m}}{\alpha L^{2}} \sinh \left[p_{e}(\omega)\right]+\cosh \left[p_{e}(\omega)\right]}\right|^{2}$,

and the discharge FTF is given by

$$
\Theta_{q}(\omega)=\frac{\alpha^{2} S_{m}^{2}}{\omega^{2}\left|S_{m}+\tilde{\varphi}(\omega)\right|^{2}}\left|\frac{\tanh \left[p_{e}(\omega)\right]}{\tanh \left[p_{e}(\omega)\right]+\frac{\alpha L^{2}}{p_{e}(\omega) T_{m}}}\right|^{2} .
$$

\section{Appendix D: Scalings}

\section{D1. Scaling of the Dual Continuum Model}

[84] The Fourier transform of the memory function for the dual continuum model $\tilde{g}\left(\omega, \tau_{i m}\right)$, given in equation (27), scales as $\left(\omega \tau_{i m}\right)^{-1 / 2}$ for $\omega \gg \tau_{i m}^{-1}$. Thus, the Fourier transform of the memory function defined in equation (29), scales as

$$
\tilde{\varphi}(\omega)=S_{i m}\left(\omega \tau_{i m}\right)^{-1 / 2}
$$

[85] We obtain the scalings of the discharge FTF discussed in section 3.2 by inserting equation (D1) in equations (C3) and (C6), for $\omega \tau_{i m} \gg 1$ and $\omega \ll \tau_{a}^{-1}$ with the activation time $\tau_{a}$ given in equation (38).

[86] The scaling of the head FTF discussed in section 3.2 are obtained by inserting equation (D1) in equations (C2) and $(\mathrm{C} 5)$, for $\omega \tau_{i m} \gg 1$. As for the homogeneous LDD model, the scaling of the head FTF for the dual continuum model, with Dirichlet BC, depends on the observation 
point $x$. Close to the discharge, that means for $x \ll L$, we can have a preasymptotic regime where the head FTF scales as $\Theta_{h}(\omega) \sim \omega^{-1 / 2}$. This scaling is obtained by considering the expansion of the hyperbolic cosine in equation (C2) for $x \ll L$ as

$$
\cosh \left[p_{e}(\omega)\left(\frac{x}{L}-1\right)\right] \simeq \cosh \left[p_{e}(\omega)\right]-p_{e}(\omega) \frac{x}{L} \sinh \left[p_{e}(\omega)\right]+\ldots
$$

and then considering the limit for $p_{e}(\omega) \gg 1$ and $\omega \ll \tau_{a}^{-1}$, with $p_{e}(\omega)$ defined previously in section $\mathrm{C} 1$.

\section{D2. Scaling of the Multicontinuum Model}

[87] We assume that the lower cutoff scale $\tau_{1}$ in equation (41) is much smaller than the upper cutoff $\tau_{2}$ so that $\tau_{1} / \tau_{2}$ $\ll 1$ and we approximate

$$
P_{\text {im }}(\tau)=\frac{1-\beta}{\tau_{2}}\left(\frac{\tau}{\tau_{2}}\right)^{-\beta} \mathcal{H}\left(\tau_{2}-\tau\right) .
$$

[88] The memory function (40) for the distribution (D3) of characteristic storage times can be written as

$$
\varphi(t)=S_{i m} \frac{1-\beta}{\tau_{2}}\left(\frac{t}{\tau_{2}}\right)^{-\beta} \int_{t / \tau_{2}}^{\infty} d z z^{\beta-1} g^{\prime}(z),
$$

where $g^{\prime}(z)$ is defined in equation (B6). The function $g^{\prime}(z)$ behaves as $z^{-1 / 2}$ for $z<1$ and decays exponentially fast for $z \gg 1$. Thus, we approximate it here by the truncated power-law distribution

$$
g^{\prime}(z)=\frac{z^{-1 / 2} \exp (-z)}{\Gamma(1 / 2)}
$$

[89] Inserting equation (D5) into equation (D4) and executing the integral for the memory function $\varphi(t)$ reads

$$
\varphi(t)=S_{i m} \frac{1-\beta}{\tau_{2} \Gamma(1 / 2)}\left(\frac{t}{\tau_{2}}\right)^{-\beta} \Gamma\left(\beta-1 / 2, t / \tau_{2}\right) .
$$

[90] This function behaves as a power-law according to $t^{-\beta}$ for $t \ll \tau_{2}$ and is cut-off exponentially fast for $t \gg \tau_{2}$. Thus, we approximate it in the following by the truncated power law (42).

[91] We obtain the scaling of the discharge FTF in the frequency regime $\tau_{2}^{-1} \ll \omega \ll \tau_{a}^{-1}$ by using equation (43) in equations (C3) and (C6), and taking the limit $\omega \tau_{2} \gg 1$. This gives straightforwardly the scalings (45) and (46).

[92] The scaling of the head FTF in the frequency regime $\tau_{2}^{-1} \ll \omega \ll \tau_{a}^{-1}$ is obtained by using equation (43) in equations (C2) and (C5). As previously discussed for the dual continuum model, the scaling of the head FTF near the discharge, for Dirichlet $\mathrm{BC}$, is obtained by expanding the hyperbolic cosine of equation $(\mathrm{C}(2))$ for $x \ll L$ and then taking the limit for $p_{e}(\omega) \gg 1$ and $\omega \ll \tau_{a}^{-1}$, with $p_{e}(\omega)$ defined in section (C1) and $\tau_{a}$ the activation time given in equation (44).

\section{Appendix E: Notation}

LD Linear Dupuit model.

LDD Linear Dupuit model with Dirichlet boundary condition at the discharge.

LDC Linear Dupuit model with Cauchy boundary at the discharge.

LR Linear reservoir model.

FTF frequency transfer function.

$d_{m}, d_{i m} \quad$ Thickness of mobile and immobile zone, respectively [L].

$f_{q} \quad$ Discharge impulse response function $\left[\mathrm{T}^{-1}\right]$.

$h, h_{0} \quad$ Hydraulic head and reference hydraulic head at outfall [L].

$h_{m}, h_{i m} \quad$ Hydraulic head of the mobile and the immobile zone [L].

$\bar{h}_{m}, \bar{h}_{i m} \quad$ Vertically averaged hydraulic head of the mobile and the immobile zone [L].

$T \quad$ Transmissivity $\left[\mathrm{L}^{2} \mathrm{~T}^{-1}\right]$.

$K_{m}, K_{i m}$ Hydraulic conductivity mobile and immobile zone $\left[\mathrm{L} \mathrm{T}^{-1}\right]$.

$L \quad$ Length of the aquifer [L].

$q \quad$ Groundwater discharge per unit aquifer surface area $\left[\mathrm{L} \mathrm{T}^{-1}\right]$.

Aquifer recharge per unit surface area $\left[\mathrm{L} \mathrm{T}^{-1}\right]$.

$\begin{array}{ll}r & \text { Aquifer rechat } \\ S & \text { Storativity. }\end{array}$

$S_{e} \quad$ Effective asymptotic storativity.

$s_{m}, s_{i m} \quad$ Specific storativity of mobile and immobile zone $\left[\mathrm{L}^{-1}\right]$.

$S_{m}, S_{i m} \quad$ Storativity of mobile and immobile zone.

$T_{m}, T_{i m}$ Transmissivity of mobile and immobile zone $\left[\mathrm{L}^{2} \mathrm{~T}^{-1}\right]$.

$\omega \quad$ Frequency $\left[\mathrm{T}^{-1}\right]$.

$x \quad$ Spatial distance from the discharge point [L].

$z$

$\alpha, \alpha$

$\beta \quad$ Exponent. Spatial distance from the bottom of the aquifer [L].

$\tau_{2} \quad$ Cut-off time [T].

$\tau_{a} \quad$ Characteristic response time of LR model [T]

$\tau_{a} \quad$ Activation time of the immobile zone in the dual and multicontinuum models [T].

$\tau_{E} \quad$ Characteristic response time of the multicontinuum model in asymptotic equilibrium [T].

$\tau_{L} \quad$ Characteristic response time of LD model [T].

$\tau_{M} \quad$ Characteristic response time of the mobile zone [T].

$\tau_{\text {im }} \quad$ Mean head diffusion time over the thickness of the immobile zone [T].

$\varphi \quad$ Memory function of the multicontinuum model $\left[\mathrm{T}^{-1}\right]$.

$\Theta_{q} \quad$ Discharge FTF.

$\Theta_{h} \quad$ Head FTF $\left[\mathrm{T}^{2}\right]$

[94] Acknowledgments. The support through the FP7 European Union Marie Curie ITN IMVUL (grant agreement 212298) is gratefully acknowledged. Tanguy Le Borgne and Joaquin Jimenez-Martinez want to express their gratitude to the project CLIMAWAT (Adapting to the Impacts of Climate Change on Groundwater Quantity and Quality), EURDF INTERREG IVA France (Channel)-England program. The comments of Monica Riva and Aldo Fiori as well as two anonymous reviewers are gratefully acknowledged. 


\section{RUSSIAN ET AL.: SCALING OF GROUNDWATER DISCHARGE}

\section{References}

Barenblatt, G., I. P. Zheltov, and I. Kochina (1960), Basic concepts in the theory of seepage of homogeneous liquids in fissured rocks [strata], PMM, 24(5), 825-864.

Bear, J. (1972), Dynamics of Fluids in Porous Media, American Elsevier, New York

Besbes, M., and G. De Marsily (1984), From infiltration to recharge: Use of a parametric transfer function, J. Hydrol., 74, 271-293.

Box, G. E. P., and G. M. Jenkins (1970), Time Series Analysis, Forecasting and Control, Holden-Day, San Francisco.

Carrera, J., X. Sánchez-Vila, I. Benet, A. Medina, G. Galarza, and J. Guimerà (1998), On matrix diffusion: Formulations, solution methods and qualitative effects, Hydrogeol. J., 6(1), 178-190, doi:10.1007/ s100400050143.

De Marsily, G. (1986), Quantitative Hydrogeology, Academic, London.

Dingman, S. L. (1994), Physical Hydrology, Prentice Hall, Englewood Cliffs, N. J.

Duffy, C. J. (2010), Dynamical modelling of concentration-age-discharge in watersheds, Hydrol. Processes, 24(12), 1711-1718, doi:10.1002/ hyp.7691.

Duffy, C. J., and J. Cusumano (1998), A low-dimensional model for concentration-discharge dynamics in groundwater stream systems, Water Resour. Res., 34(9), 2235-2247, doi:10.1029/98WR01705.

Duffy, C. J., and L. W. Gelhar (1985), A frequency domain approach to water quality modeling in groundwater: Theory, Water Resour., 21(8), 1175-1184

Duffy, C. J., and D.-H. Lee (1992), Base flow response from nonpoint source contamination: Simulated spatial variability in source, structure, and initial condition, Water Resour. Res., 28(3), 905-914.

Dykhuizen, C. R. (1987), Transport of solutes through unsaturated fractured media, Water Resour., 21(12), 1531-1539.

Dykhuizen, R. C. (1990), New coupling term for dual porosity models, Water Resour. Res., 26(2), 351-356, doi:10.1016/0148-9062(90)92852-

Erskine, A. D., and A. Papaioannou (1997), The use of aquifer response rate in the assessment of groundwater resources, J. Hydrol., 202, 373391.

Fiori, A., and D. Russo (2007), Numerical analyses of subsurface flow in a steep hillslope under rainfall: The role of the spatial heterogeneity of the formation hydraulic properties, Water Resour. Res., 43, W07445, doi: 10.1029/2006WR005365.

Fiori, A., D. Russo, and M. Di Lazzaro (2009), Stochastic analysis of transport in hillslopes: Travel time distribution and source zone dispersion, Water Resour. Res., 45, W08435, doi:10.1029/2008WR007668.

Freeze, R. A. (1975), A stochastic-conceptual analysis of one-dimensional groundwater flow in nonuniform homogeneous media, Water Resour. Res., 11(5), 725-741.

Gelhar, L. (1974), Stochastic analysis of phreatic aquifers, Water Resour. Res., 10(3), 539-545.

Gelhar, L. W., and J. L. Wilson (1974), Ground-water quality modeling, Ground Water, 12(6), 399-408.

Haggerty, R., and S. M. Gorelick (1995), Multiple-rate mass transfer for modeling diffusion and surface reactions in media with pore-scale heterogeneity, Water Resour. Res., 31(10), 2383-2400.

Hurst, H. E. (1951), Long-term storage capacity of reservoirs, Trans. Am. Soc. Civ. Eng., 116, 770-808.

Jiménez-Martínez, J., L. Longuevergne, T. L. Borgne, P. Davy, A. Russian, and O. Bour (2013), A frequency domain analysis to characterize heterogeneity and recharge mechanisms in fractured crystalline-rock aquifers, Water Resour. Res., 49, doi:10.1002/wrcr.20260.

Juki, D., and V. Denijuki (2004), A frequency domain approach to groundwater recharge estimation in karst, J. Hydrol., 289(1-4), 95-110, doi: 10.1016/j.jhydrol.2003.11.005.
Kantelhardt, J. W., D. Rybski, S. a. Zschiegner, P. Braun, E. KoscielnyBunde, V. Livina, S. Havlin, and A. Bunde (2003), Multifractality of river runoff and precipitation: Comparison of fluctuation analysis and wavelet methods, Phys. A: Stat. Mech. Appl., 330(1-2), 240-245, doi: 10.1016/j.physa.2003.08.019.

Kantelhardt, J. W., E. Koscielny-Bunde, D. Rybski, P. Braun, A. Bunde, and S. Havlin (2006), Long-term persistence and multifractality of precipitation and river runoff records, J. Geophys. Res., 111, D01106, doi : 10.1029/2005JD005881.

Koscielny-Bunde, E., J. W. Kantelhardt, P. Braun, A. Bunde, and S. Havlin (2006), Long-term persistence and multifractality of river runoff records : Detrended fluctuation studies, J. Hydrol., 322(1-4), 120-137, doi: 10.1016/j.jhydrol.2005.03.004

Labat, D., A. Mangin, and R. Ababou (2002), Rainfall-runoff relations for karstic springs: Multifractal analyses, J. Hydrol., 256, 176-195.

Labat, D., J. Masbou, E. Beaulieu, and A. Mangin (2011), Scaling behavior of the fluctuations in stream flow at the outlet of karstic watersheds, France, J. Hydrol., 410(3-4), 162-168, doi:10.1016/j.jhydrol.2011.09.010.

Little, M. A., and J. P. Bloomfield (2010), Robust evidence for random fractal scaling of groundwater levels in unconfined aquifers, J. Hydrol., 393(3-4), 362-369, doi:10.1016/j.jhydrol.2010.08.031

Livina, V., Z. Kizner, P. Braun, T. Molnar, a. Bunde, and S. Havlin (2007), Temporal scaling comparison of real hydrological data and model runoff records, J. Hydrol., 336(1-2), 186-198, doi:10.1016/j.jhydrol.2007.01.014.

Manga, M. (1999), On the timescales characterizing groundwater discharge at springs, $J$. Hydrol., 219(1-2), 56-69, doi:10.1016/S00221694(99)00044-X.

McGuire, K. J. (2006), A review and evaluation of catchment transit time modeling, J. Hydrol., 330(3-4), 543-563.

Molenat, J., P. Davy, C. Gascuelodoux, and P. Durand (1999), Study of three subsurface hydrologic systems based on spectral and cross-spectral analysis of time series, J. Hydrol., 222(1-4), 152-164, doi:10.1016/ S0022-1694(99)00107-9.

Molenat, J., P. Davy, and P. Durand (2000), Spectral and cross-spectral analysis of three hydrological systems, Phys. Chem. Earth, Part B, 25(4), 391-397.

Nash, J. E. (1957), The Form of the Instantaneous Unit Hydrograph, C. R. et Rapports, Toronto, 3-14 Sept.

Nasri, S., C. Cudennec, J. Albergel, and R. Berndtsson (2004), Use of a geomorphological transfer function to model design floods in small hillside catchments in semiarid Tunisia, J. Hydrol., 287(1-4), 197-213.

Scanlon, B. R., R. W. Healy, and P. G. Cook (2002), Choosing appropriate techniques for quantifying groundwater recharge, Hydrogeol. J., 10, 18-39, doi:10.1007/s10040-0010176-2.

Tessier, Y., D. Schertzer, P. Hubert, and S. Pecknoldl (1996), Multifractal analysis and modeling of rainfall and river flows and scaling, causal transfer functions, J. Geophys. Res., 101(D21), 26,427-26,440

Turcotte, D. L., and L. Greene (1993), A scale-invariant approach to floodfrequency analysis, Stochastic Hydrol. Hydraul., 7(1), 33-40, doi: 10.1007/BF01581565

Warren, J. E., and P. Root (1963), The behavior of naturally fractured reservoirs, Soc. Pet. Eng. J., 3(3), 245-255.

Wiener, N. (1949), Extrapolation, Interpolation, and Smoothing of Stationary Time Series, MIT Press, New York.

Zhang, Y.-K. (2004), Temporal scaling of hydraulic head and river base flow and its implication for groundwater recharge, Water Resour. Res., 40, W03504, doi:10.1029/2003WR002094.

Zhang, Y.-K. (2005), Temporal scaling of hydraulic head fluctuations: Nonstationary spectral analyses and numerical simulations, Water Resour. Res., 41, W07031, doi:10.1029/2004WR003797.

Zhang, Y.-K., and X. Yang (2010), Effects of variations of river stage and hydraulic conductivity on temporal scaling of groundwater levels: numerical simulations, Stochastic Environ. Res. Risk Assess., 24(7), 1043-1052, doi:10.1007/s00477-010-0437-5. 